\title{
First-Principles Study on Influences of Crystal Structure and Orientation on Band Offsets at the $\mathrm{CdS} / \mathrm{Cu}_{2} \mathrm{ZnSnS}_{4}$ Interface
}

\author{
Wujisiguleng Bao and Masaya Ichimura \\ Department of Engineering Physics, Electronics and Mechanics, Nagoya Institute of Technology, Gokiso, Showa-Ku, \\ Nagoya 466-8555, Japan \\ Correspondence should be addressed to Masaya Ichimura, ichimura.masaya@nitech.ac.jp
}

Received 20 July 2012; Accepted 9 November 2012

Academic Editor: Mahmoud M. El-Nahass

Copyright (๑) 2012 W. Bao and M. Ichimura. This is an open access article distributed under the Creative Commons Attribution License, which permits unrestricted use, distribution, and reproduction in any medium, provided the original work is properly cited.

$\mathrm{Cu}_{2} \mathrm{ZnSnS}_{4}$ (CZTS) has attracted much attention recently as an absorber layer material in a heterojunction solar cell. Using the first-principles method, we calculate the band offsets for the CdS/CZTS heterojunction. The valence band offset $\Delta E_{v}$ is $1.2 \mathrm{eV}$ for the (001) CdS/CZTS heterointerface and $1.0 \mathrm{eV}$ for the (010) heterointerface, when CZTS is considered to crystallize in the kesterite structure. When CZTS is considered to crystallize in the stannite structure, $\Delta E_{v}=1.1 \mathrm{eV}$ for the (001) heterointerface and $\Delta E_{v}=1.3 \mathrm{eV}$ for the (010) heterointerface. In any case, the conduction band minimum of CZTS is higher than that of CdS, and the conduction band offset $\Delta E_{c}$ is in a range between 0.1 and $0.4 \mathrm{eV}$.

\section{Introduction}

$\mathrm{Cu}_{2} \mathrm{ZnSnS}_{4}$ (CZTS) is a p-type semiconductor with a band gap of $1.5 \mathrm{eV}$, which is close to the optimum band gap for solar cells and has high absorption coefficient $\left(>10^{4} \mathrm{~cm}^{-1}\right)$ in the visible region. In addition, the earth abundance and the nontoxic nature of the elements eliminate environmental concerns. Thus, CZTS has attracted much attention recently as an absorber layer material in a heterojunction solar cell $[1,2]$. So far, CdS has been selected as a partner of the heterojunction $[2,3]$. CdS is an n-type semiconductor with a band gap of $2.4 \mathrm{eV}$ and has also been used for $\mathrm{CuInSe}_{2}$ and CdTe-based solar cells. The solar cells based on CZTS absorber layers and CdS window layers have been developed rapidly, and the highest efficiency obtained so far is about $8.4 \%[4]$.

Electrical transport properties of heterojunction solar cells are greatly influenced by the band offsets at the heterointerface. The difference in the band gap across the interface is divided into the valence band and the conduction band offsets. These offsets may dramatically influence both the photocurrent and dark current of heterojunction solar cells. The valence band offset for CdS/CZTS has been studied both theoretically and experimentally. Chen et al. obtained the valence band offset $\Delta E_{v}$ of $1.01 \mathrm{eV}$ on the basis of the firstprinciples calculation [5]. On the other hand, Nagoya et al. obtained a significantly smaller value of valence band offset, $0.3 \sim 0.57 \mathrm{eV}$ on the basis of the first-principles calculation, considering that the lattice of CdS is strained to fit the CZTS lattice [6]. Haight et al. experimentally obtained a similar value, $0.54 \mathrm{eV}$, using ultraviolet photoelectron spectroscopy (UPS) [7]. If $\Delta E_{v}=1.01 \mathrm{eV}$, the conduction band minimum (CBM) of CZTS is higher than that of CdS; that is, the band alignment is of type II. On the other hand, if $\Delta E_{v}=$ $0.54 \mathrm{eV}, \mathrm{CBM}$ of CZTS is lower than that of CdS and the band alignment is of type I. In a previous work [8], we also carried out the first-principles calculation for the CdS/CZTS heterostructure and obtained $\Delta E_{v}$ of $1.2 \mathrm{eV}$, similar to the results of Chen et al. In the previous calculation, we assumed that the heterointerface is (001) oriented and that CZTS crystallizes in the kesterite structure. It is generally believed that the kesterite is the most stable structure for CZTS, but several theoretical calculations predict that the energy difference from the stannite structure is small compared with thermal energy at the material growth temperature $[9,10]$. Thus, we may expect that the actual CZTS film is a mixture of 


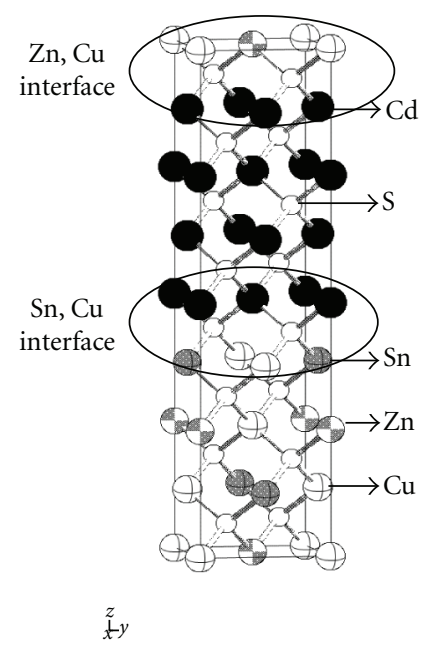

(a)

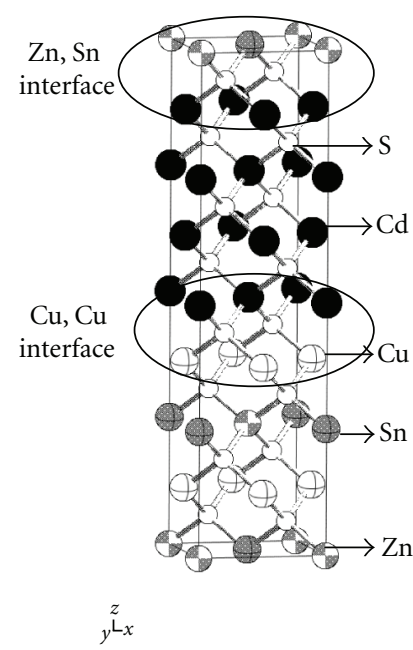

(b)

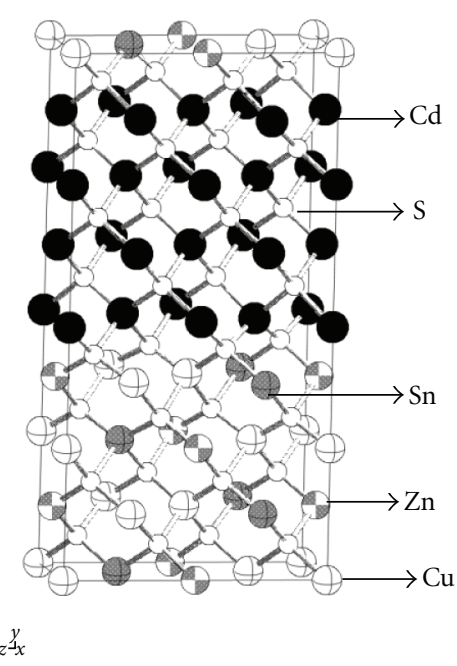

(c)

Figure 1: (a) Supercell of (001)-oriented CdS/kesterite-CZTS. (b) Supercell of (001)-oriented CdS/stannite-CZTS. (c) Supercell of (010)oriented CdS/kesterite-CZTS.

the kesterite and stannite phases. In addition, both the CZTS and CdS films in the solar cell are in fact polycrystalline, and thus the interfaces of various different crystallographic orientations should be formed. Therefore, in this study, we attempt to predict the influences of crystal structure and interface orientation on the band offset by calculating the band offsets for both the kesterite and stannite structures with (001) and (010) interface orientations.

\section{Calculation}

Figure 1 shows the supercells of CdS/CZTS heterojunctions considered in this study. CdS is assumed to have the cubic crystal structure. Figure 1(a) shows the (001) CdS/kesteriteCZTS supercell. In the supercell along the (001) orientation, two unit cells of CdS and one unit cell of CZTS are stacked alternately. There are two inequivalent interfaces between CdS and CZTS, and the ( $\mathrm{Cu}, \mathrm{Zn})$ layer in CZTS is adjacent to one interface, while the $(\mathrm{Cu}, \mathrm{Sn})$ layer is adjacent to the other interface. Figure 1(b) shows the (001) CdS/stanniteCZTS supercell. The ( $\mathrm{Sn}, \mathrm{Zn}$ ) layer of CZTS is adjacent to one interface and the $(\mathrm{Cu}, \mathrm{Cu})$ layer is adjacent to the other interface. Figure 1(c) shows the (010) supercell of $\mathrm{CdS} /$ kesterite-CZTS. The two atomic layers in CZTS adjacent to the interfaces contain the same number of atoms $(2 \mathrm{Cu}$, $\mathrm{Zn}$, and $\mathrm{Sn}$ ), and thus the two interfaces are considered to be effectively equivalent. The two interfaces in the (010) CdS/stannite-CZTS (not shown here) are also considered to be equivalent.

In the calculation, the average value of lattice constants of CdS and CZTS bulk materials was used for the lattice constant along the heterointerface. The lattice spacing in the perpendicular direction was assumed to be the same as in the respective bulk material. The CZTS and CdS layers in the superstructure are considered to simulate the respective bulk material. Thus, the position of each atom was not optimized; that is, the relative positions of atoms in the CdS and CZTS unit cells are the same as in the bulk material. On the other hand, the $\mathrm{S}$ atoms at the interface were allowed to move to minimize the total energy.

The calculation of density of states (DOS) was performed on the basis of the first-principles, density-functional, pseudopotential method using the PHASE code developed by Institute of Industrial Science, University of Tokyo [11]. The generalized gradient approximation (GGA) is developed by Perdew-Burke-Ernzerhof exchange-correlation energy [12].

We first calculated the energy level difference between the valence band maximum (VBM) and reference core levels for CdS and CZTS then calculated the core levels for the CdS/CZTS supercell. The band offsets are given by $[13,14]$

$$
\begin{gathered}
\Delta E_{v}=\Delta E_{\mathrm{VBM}-1}^{\mathrm{CZTS}}-\Delta E_{\mathrm{VBM}-2}^{\mathrm{CdS}}-\Delta E_{\mathrm{core}}^{\mathrm{CZTS} / \mathrm{CdS}}, \\
\Delta E_{c}=\left|\Delta E_{g}-\Delta E_{v}\right|,
\end{gathered}
$$

where $\Delta E_{\mathrm{VBM}-1}^{\mathrm{CZTS}}\left(\Delta E_{\mathrm{VBM}-2}^{\mathrm{CdS}}\right)$ is the energy separation between the reference core levels and VBM for the CZTS (CdS) bulk, and $\Delta E_{\text {core }}^{\text {CZTS/CdS }}$ is the difference in the core level in the CZTS/CdS supercell. This equation is based on the idea that the energy difference between the core level and VBM in the respective bulk material is conserved in the heterostructure. $\Delta E_{g}$ is the band gap difference between CdS and CZTS. Since the band gap is generally underestimated in the calculation based on GGA, we used the experimentally determined band gap values (2.4 eV for CdS and $1.5 \mathrm{eV}$ for CZTS).

The energy separation between VBM and the reference core level is obtained by calculating the DOS for CZTS and CdS. For CZTS, the S 3s, Sn 4d, and Zn 3d energy levels form a narrow band well separated from the upper continuous valence bands as shown in Figure 2(a) and thus may be regarded as the reference core level. For CdS, the S 3s and Cd $4 \mathrm{~d}$ levels may be regarded as the reference core level, as shown in Figure 2(b). Thus, there are several different choices of 


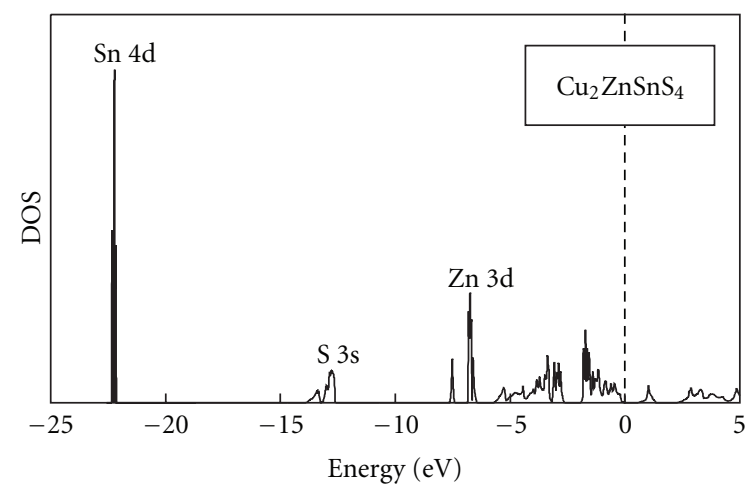

(a)

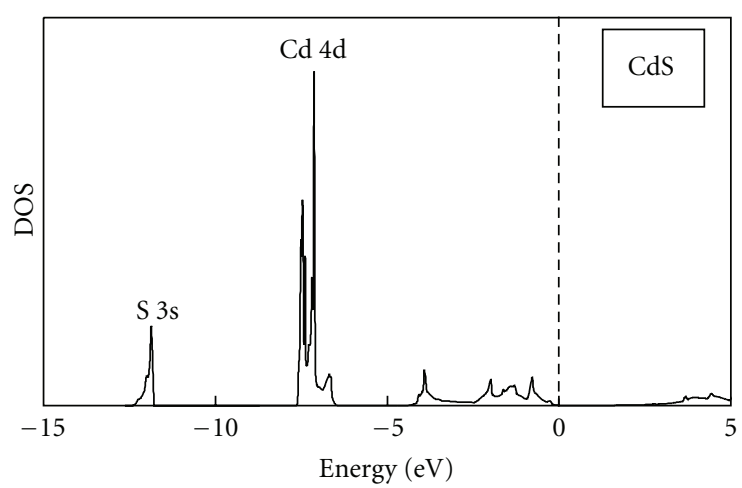

(b)

Figure 2: Density of states for (a) $\mathrm{Cu}_{2} \mathrm{ZnSnS}_{4}$ and (b) CdS.

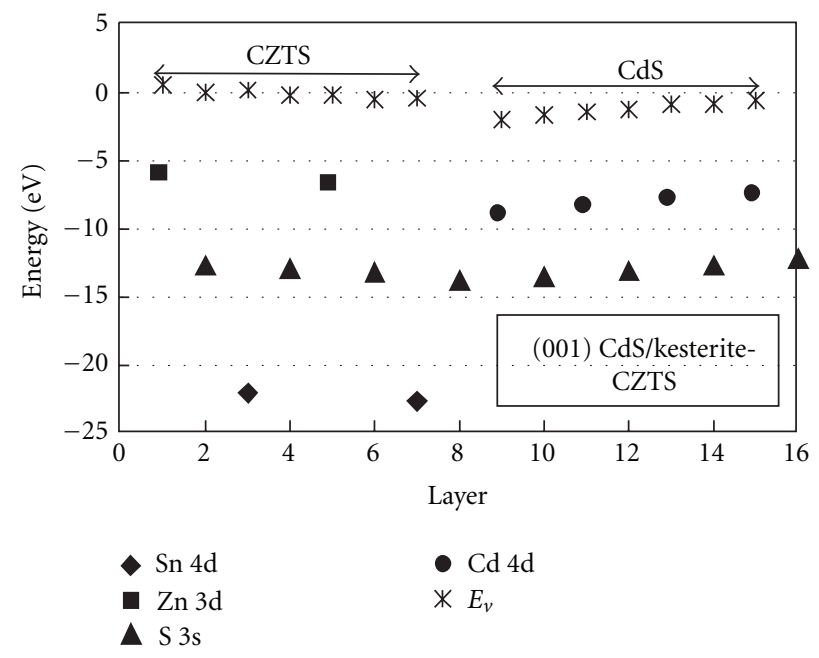

(a)

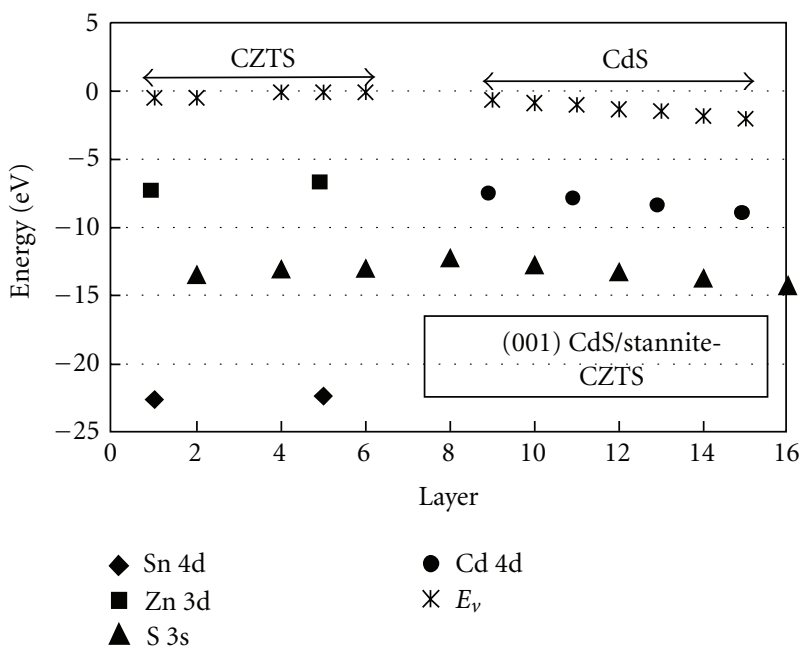

(b)

Figure 3: Calculated reference core levels and VBM for the respective layer in the (001)-oriented CdS/CZTS supercell. (a) CdS/kesteriteCZTS. (b) CdS/stannite-CZTS.

the core levels in the above equation, and all of them are examined in the calculation. Both CZTS and CdS have the same anion $S$, and the $S 3 p$ orbital composes the VBM states. The energy of VBM relative to $\mathrm{S} 3 \mathrm{~s}$ is $12.8 \mathrm{eV}$ in CZTS and $11.9 \mathrm{eV}$ in CdS. The larger separation between the $\mathrm{S} 3 \mathrm{~s}$ level and VBM in CZTS is caused by the repulsion between the $S$ $3 p$ and $\mathrm{Cu} 3 \mathrm{~d}$ states, which moves VBM upwards [15].

\section{Results and Discussion}

The calculated energies of the reference core levels and obtained VBM for the respective layer in the CdS/CZTS supercell are shown in Figure 3. Figure 3(a) shows the results of (001) CdS/kesterite-CZTS. Layer 0 (or 16) corresponds to the $\mathrm{S}$ layer at the $(\mathrm{Zn}, \mathrm{Cu})$ interface, and layer 8 corresponds to the $\mathrm{S}$ layer at the $(\mathrm{Sn}, \mathrm{Cu})$ interface. As shown in this figure, the energy level of a certain kind of atom orbital is not constant but dependent on the position (layer number). Polarization field due to the different atom configurations in the two interface layers, as discussed below, may cause this. As noted above, (1) implies that the energy difference between VBM and each reference level remains the same as in the respective compound. The " $E_{v}$ " energies denoted by the stars in Figure 3(a) show the predicted VBM positions for both the CdS and CZTS layers. The VBM position of layer 1 was obtained based on the $\mathrm{Zn} 3 \mathrm{~d}$ level in that layer, and the VBM position of layer 2 based on the $S 3$ s level, and so on. As noted above, there are two inequivalent interfaces, and for the $(\mathrm{Zn}, \mathrm{Cu})$ interface, the valence band offset was estimated as $\Delta E_{v}=1.1 \mathrm{eV}$ from the VBM positions at layer 1 and layer 15 . For the ( $\mathrm{Sn}, \mathrm{Cu})$ interface, $\Delta E_{v}$ was estimated as $\Delta E_{v}=1.6 \mathrm{eV}$ from the VBM positions of layer 7 and layer 9 . The corresponding conduction band offsets are $\Delta E_{c}=0.2 \mathrm{eV}$ for the $(\mathrm{Zn}, \mathrm{Cu})$ interface and $\Delta E_{c}=0.7 \mathrm{eV}$ for the $(\mathrm{Sn}$, $\mathrm{Cu}$ ) interface. If the VBM energy is averaged within $\mathrm{CdS}$ and CZTS parts, then $\Delta E_{v}=1.2 \mathrm{eV}$ and $\Delta E_{c}=0.3 \mathrm{eV}$.

The results for the (001) CdS/stannite-CZTS structure are shown in Figure 3(b). Layer 0 (or 16) corresponds to 


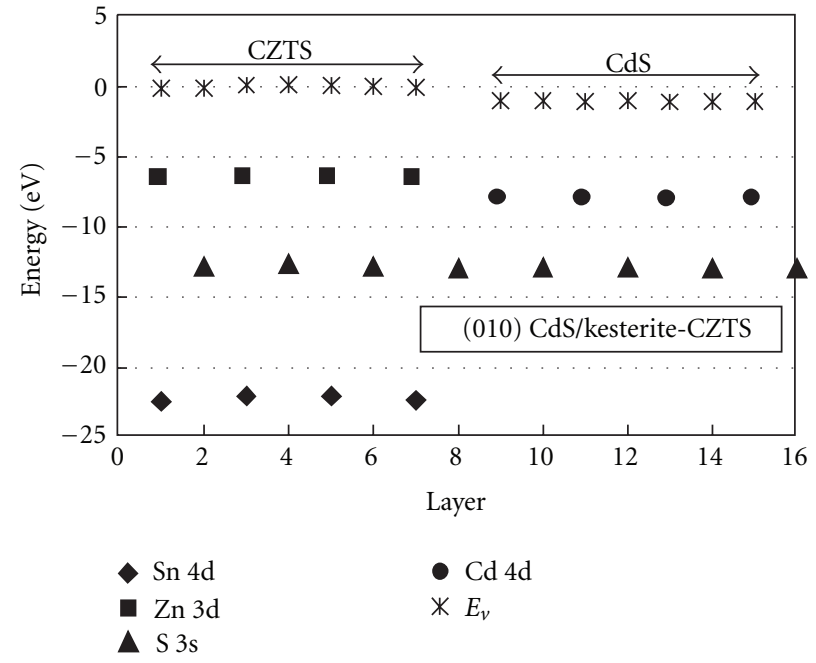

(a)

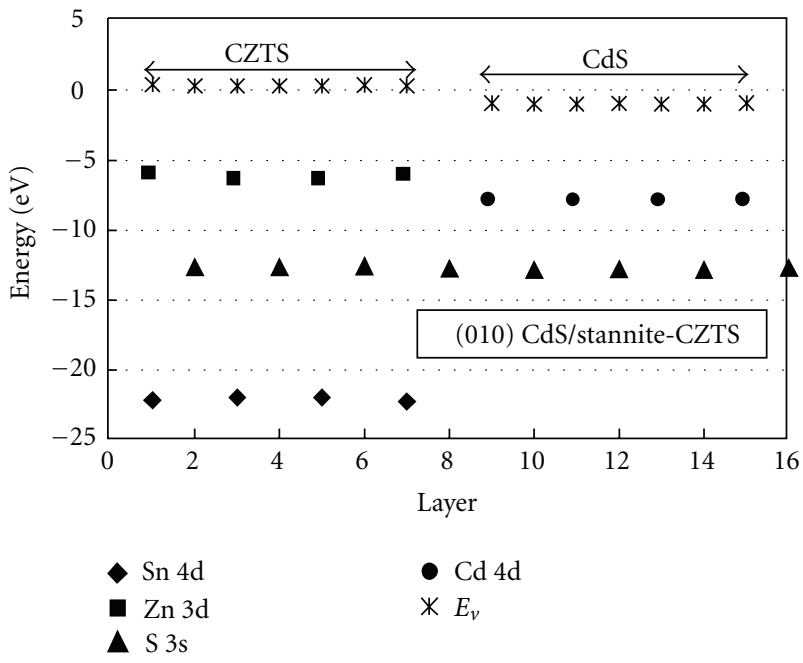

(b)

FIGURE 4: Calculated reference core levels and VBM for the respective layer in the (010)-oriented CdS/CZTS supercell. (a) CdS/kesteriteCZTS. (b) CdS/stannite-CZTS.

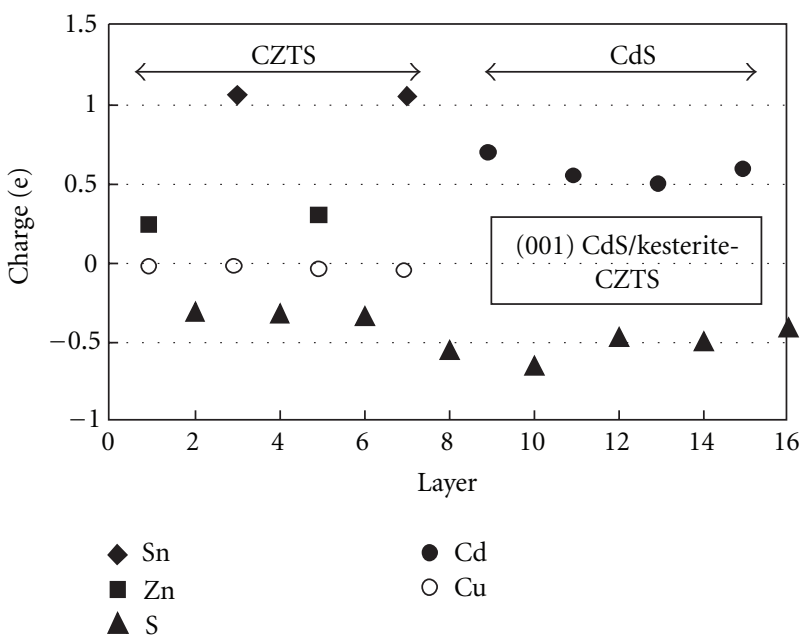

(a)

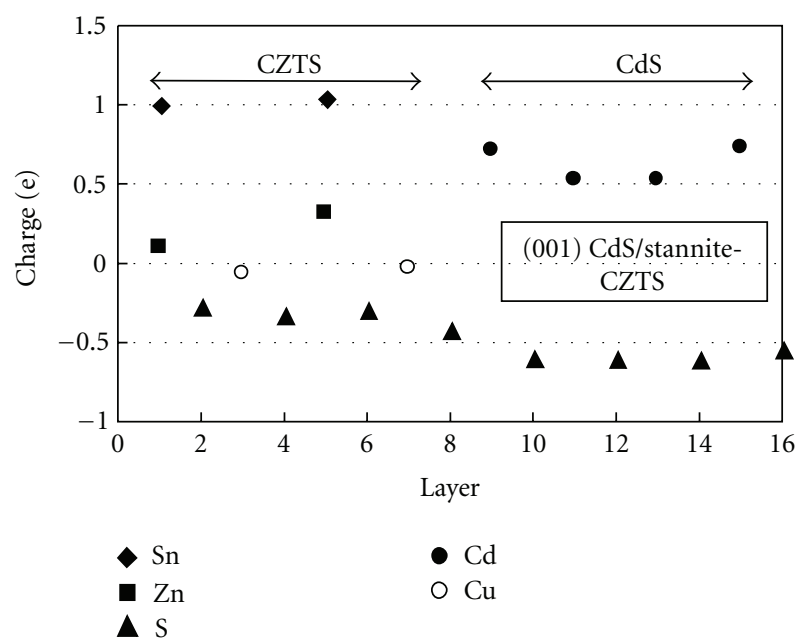

(b)

FIGURE 5: Obtained charge of each atom in the (001)-oriented supercell. (a) CdS/kesterite-CZTS. (b) CdS/stannite-CZTS.

the $\mathrm{S}$ layers at the $(\mathrm{Zn}, \mathrm{Sn})$ interface and layer 8 to the $\mathrm{S}$ layer at the $(\mathrm{Cu}, \mathrm{Cu})$ interface. As in Figure 3(a), the energy level of a certain kind of atom orbital is dependent on the position (layer number). The energy level tends to be lower near the ( $\mathrm{Zn}, \mathrm{Sn}$ ) interface (layer 0 or 16). The valence band offset was estimated as $\Delta E_{v}=1.5 \mathrm{eV}$ for the (Zn, Sn) interface and $\Delta E_{v}=0.6 \mathrm{eV}$ for the $(\mathrm{Cu}, \mathrm{Cu})$ interface. The corresponding conduction band offsets are $\Delta E_{c}=0.6 \mathrm{eV}$ for the ( $\mathrm{Zn}, \mathrm{Sn}$ ) interface and $\Delta E_{c}=-0.3 \mathrm{eV}$ for the $(\mathrm{Cu}, \mathrm{Cu})$ interface. If the VBM energy is averaged within each parts, then $\Delta E_{v}=1.1 \mathrm{eV}$ and $\Delta E_{c}=0.2 \mathrm{eV}$.

The results for (010) CdS/kesterite-CZTS are shown in Figure 4(a). In contrast to the (001) heterostructures (Figure 3 ), the energy level of each atom orbital is only weakly dependent on the layer number. As noted above, in the (010) structure, the two interfaces are effectively equivalent, and thus no polarization field is formed in each layer. The VBM energy is averaged within each parts, the band offsets are estimated as $\Delta E_{v}=1.0 \mathrm{eV}$ and $\Delta E_{c}=0.1 \mathrm{eV}$. Figure $4(\mathrm{~b})$ shows the results for (010) CdS/stannite-CZTS. The obtained band offsets are $\Delta E_{v}=1.3 \mathrm{eV}$ and $\Delta E_{c}=0.4 \mathrm{eV}$.

We obtained the charge of each atom for the respective layers in the (001) supercells of both kesterite and stannite CZTS, to discuss the position dependence of the core levels. The Sn atom has the largest positive charge among the constituent atoms. In the (001) CdS/kesterite-CZTS structure, the net charge is more positive near the $(\mathrm{Sn}, \mathrm{Cu})$ interface than near the $(\mathrm{Zn}, \mathrm{Cu})$ interface as shown in Figure 5(a), which leads to lowering of the VBM position near the (Sn, $\mathrm{Cu}$ ) interface (layer 8). For the (001) CdS/stannite-CZTS structure, the net charge is more positive near the $(\mathrm{Zn}, \mathrm{Sn})$ interface than near the $(\mathrm{Cu}, \mathrm{Cu})$ interface, and thus the VBM 


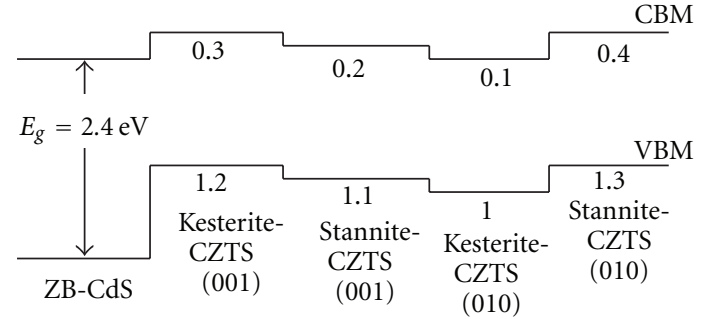

FIGURE 6: Band alignments of CdS and different crystal structures of CZTS (kesterite and stannite) with (001) and (010) orientations.

position is lower near the ( $\mathrm{Zn}, \mathrm{Sn}$ ) interface (layer 0 or 16 ). The difference in $\Delta E_{v}$ between the two interfaces may be caused by electric dipole at the interface [16]. For the (001) supercells, since the two interfaces are not equivalent, the dipole charge may be different between the two interfaces. However, we are unable to identify the dipole charge in the analysis so far.

The band alignments obtained in this work are summarized in Figure 6. The obtained $\Delta E_{v}$ values are in a range of $1.0 \mathrm{eV} \sim 1.3 \mathrm{eV}$ and $\Delta E_{c} 0.1 \mathrm{eV} \sim 0.4 \mathrm{eV}$. Thus, there will be some difference in the band offsets between the different crystal structures and the different orientations. In addition, for a certain crystal structure and orientation, the band offsets may be different depending on the local atom arrangement at the interface, as can be seen from the difference in $\Delta E_{v}$ between the two inequivalent interfaces in Figures 3(a) and 3(b). Therefore, the local band offset may fluctuate in a CdS/CZTS heterostructure. However, in any case, CBM of CZTS is higher than that of CdS; that is, the CdS/CZTS heterojunction is of type II. Therefore, there will be no barrier for the flow of photogenerated electrons from CZTS to CdS, and thus the quantum efficiency can potentially be high. However, since VBM of CZTS is close to $\mathrm{CBM}$ of CdS, the recombination probability of majority carriers will be high, which will lead to reduced output voltage.

\section{Conclusion}

We have calculated the band offsets for the CdS/CZTS heterojunction by using the first-principles, density-functional, pseudopotential method for two different crystal structures of CZTS (kesterite and stannite) and two different interface orientations ((001) and (010)). For CdS/kesterite-CZTS, the obtained valence band offset $\Delta E_{v}=1.2 \mathrm{eV}$ for the (001) orientation and $\Delta E_{v}=1.0 \mathrm{eV}$ for the (010) orientation. For CdS/stannite-CZTS, $\Delta E_{v}=1.1 \mathrm{eV}$ for the (001) orientation and $\Delta E_{v}=1.3 \mathrm{eV}$ for the (010) orientation. In any case, the CdS/CZTS heterojunction is of type II; that is, CBM of CZTS is higher than that of CdS.

\section{References}

[1] K. Ito and T. Nakazawa, "Electrical and optical properties of stannite-type quaternary semiconductor thin films," Japanese Journal of Applied Physics, vol. 27, no. 11, pp. 2094-2097, 1988.
[2] H. Katagiri, " $\mathrm{Cu}_{2} \mathrm{ZnSnS}_{4}$ thin film solar cells," Thin Solid Films, vol. 480-481, pp. 426-432, 2005.

[3] D. B. Mitzi, O. Gunawan, T. K. Todorov, K. Wang, and S. Guha, "The path towards a high-performance solution-processed kesterite solar cell," Solar Energy Materials and Solar Cells, vol. 95, no. 6, pp. 1421-1436, 2011.

[4] B. Shin, O. Gunawan, Y. Zhu, N. A. Bojarczuk, S. Jay Chey, and S. Guha, "Thin film solar cell with $8.4 \%$ power conversion efficiency using an earth-abundant $\mathrm{Cu}_{2} \mathrm{ZnSnS}_{4}$ absorber," Progress in Photovoltaics. In press.

[5] S. Chen, A. Walsh, J. H. Yang et al., "Compositional dependence of structural and electronic properties of $\mathrm{Cu}_{2} \mathrm{ZnSn}(\mathrm{S}, \mathrm{Se})_{4}$ alloys for thin film solar cells," Physical Review B, vol. 83, no. 12, Article ID 125201, 2011.

[6] A. Nagoya, R. Asahi, and G. Kresse, "first-principles study of $\mathrm{Cu}_{2} \mathrm{ZnSnS}_{4}$ and the related band offsets for photovoltaic applications," Journal of Physics, vol. 23, no. 40, Article ID 404203, 2011.

[7] R. Haight, A. Barkhouse, O. Gunawan et al., "Band alignment at the $\mathrm{Cu}_{2} \mathrm{ZnSn}\left(\mathrm{S}_{x} \mathrm{Se}_{1-x}\right)_{4} / \mathrm{CdS}$ interface," Applied Physics Letters, vol. 98, no. 25, Article ID 253502, 2011.

[8] W. Bao and M. Ichimura, "Prediction of the band offsets at the $\mathrm{CdS} / \mathrm{Cu}_{2} \mathrm{ZnSnS}_{4}$ interface based on the first-principles calculation," Japanese Journal of Applied Physics, vol. 51, no. 10, pp. 10NC31-10NC314, 2012.

[9] M. Ichimura and Y. Nakashima, "Analysis of atomic and electronic structures of $\mathrm{Cu}_{2} \mathrm{ZnSnS}_{4}$ based on first-principle calculation," Japanese Journal of Applied Physics, vol. 48, no. 9, Article ID 090202, 2009.

[10] S. Chen, X. G. Gong, A. Walsh, and S. H. Wei, "Crystal and electronic band structure of $\mathrm{Cu}_{2} \mathrm{ZnSnX}_{4} \quad(\mathrm{X}=\mathrm{S}$ and Se) photovoltaic absorbers: first-principles insights," Applied Physics Letters, vol. 94, no. 4, Article ID 041903, 2009.

[11] http://www.ciss.iis.u-tokyo.ac.jp/rss21/en/index.html.

[12] J. P. Perdew, K. Burke, and M. Ernzerhof, "Generalized gradient approximation made simple," Physical Review Letters, vol. 77, no. 18, pp. 3865-3868, 1996.

[13] A. J. Nelson, C. R. Schwerdtfeger, S.-H. Wei et al., "Theoretical and experimental studies of the $\mathrm{ZnSe} / \mathrm{CuInSe} \mathrm{S}_{2}$ heterojunction band offset," Applied Physics Letters, vol. 62, no. 20, p. 2557, 1993.

[14] S. H. Wei and A. Zunger, "Band offsets at the CdS/CuInSe heterojunction," Applied Physics Letters, vol. 63, no. 18, p. 2549, 1993.

[15] S. H. Wei and A. Zunger, "Calculated natural band offsets of all II-VI and III-V semiconductors: chemical trends and the role of cation d orbitals," Applied Physics Letters, vol. 72, no. 16, pp. 2011-2013, 1998.

[16] S. Picozzi, A. Continenza, and A. J. Freeman, "Electric fields and valence-band offsets at strained [111] heterojunctions," Physical Review B, vol. 55, no. 19, pp. 13080-13087, 1997. 


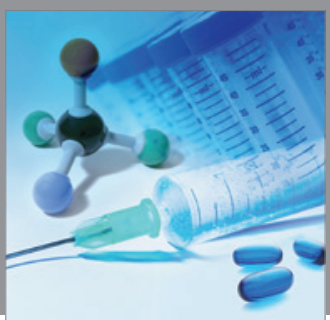

International Journal of

Medicinal Chemistry

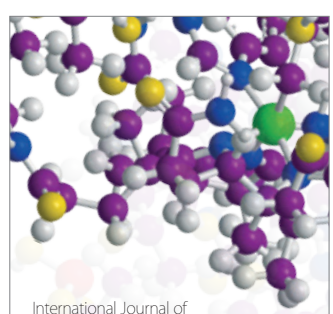

Carbohydrate Chemistry

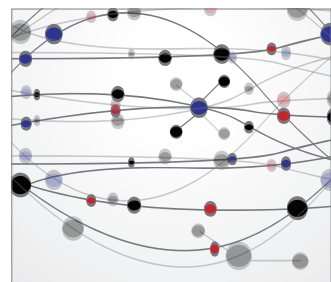

The Scientific World Journal
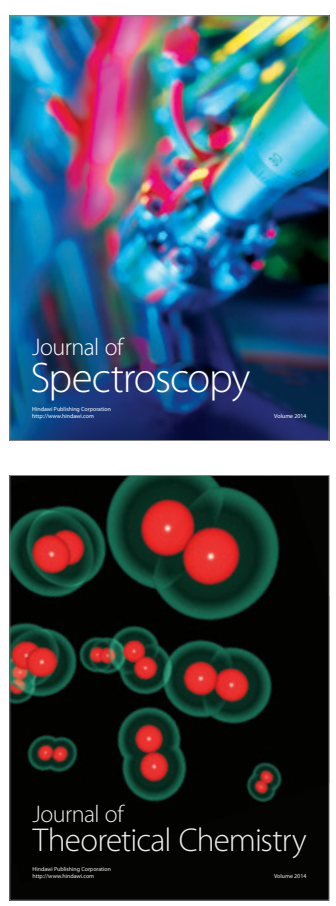
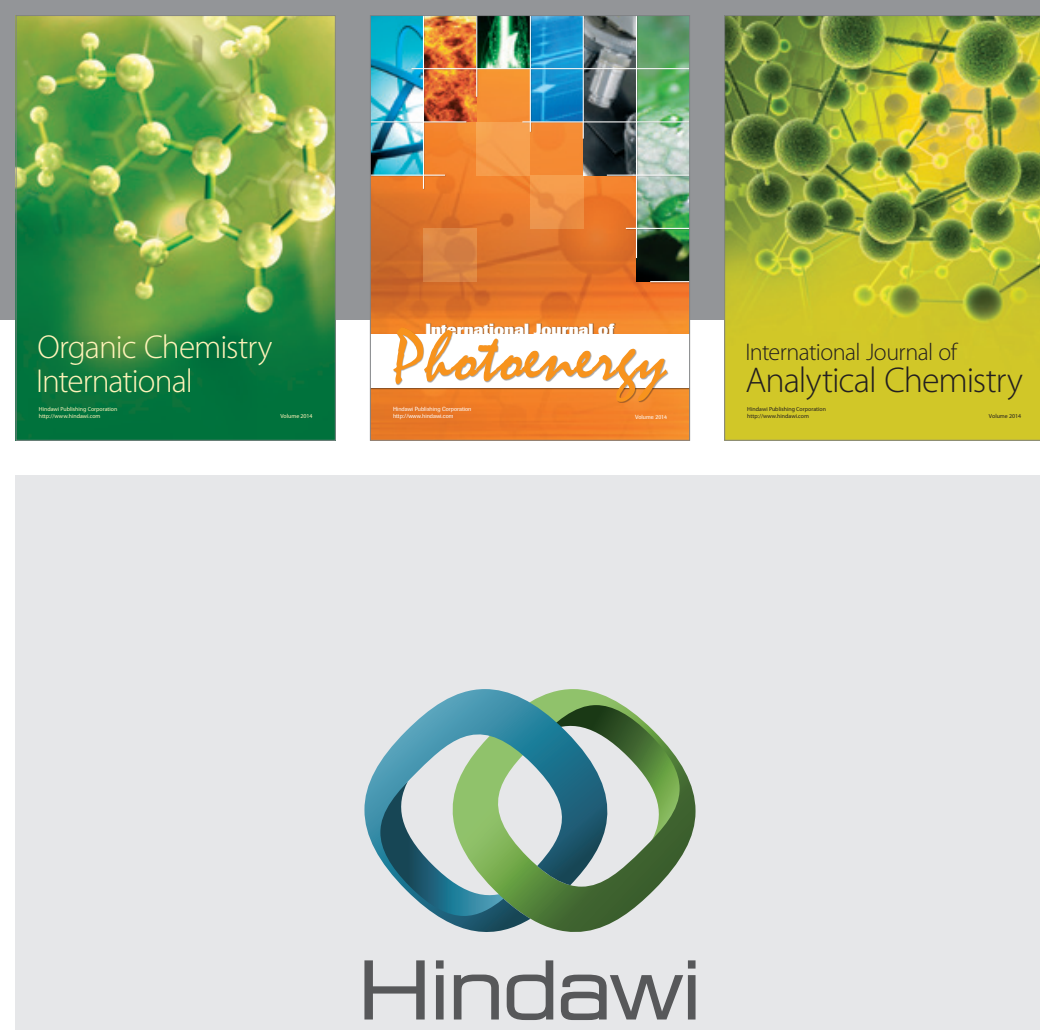

Submit your manuscripts at

http://www.hindawi.com
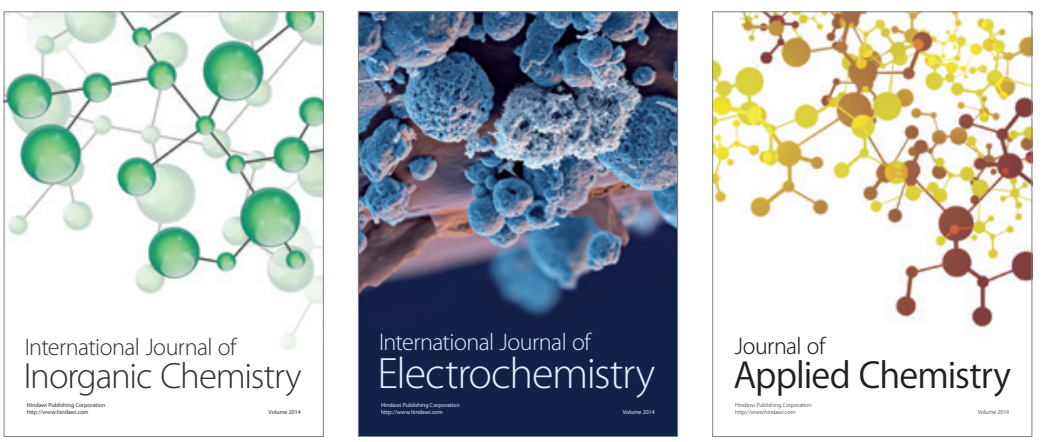

Journal of

Applied Chemistry
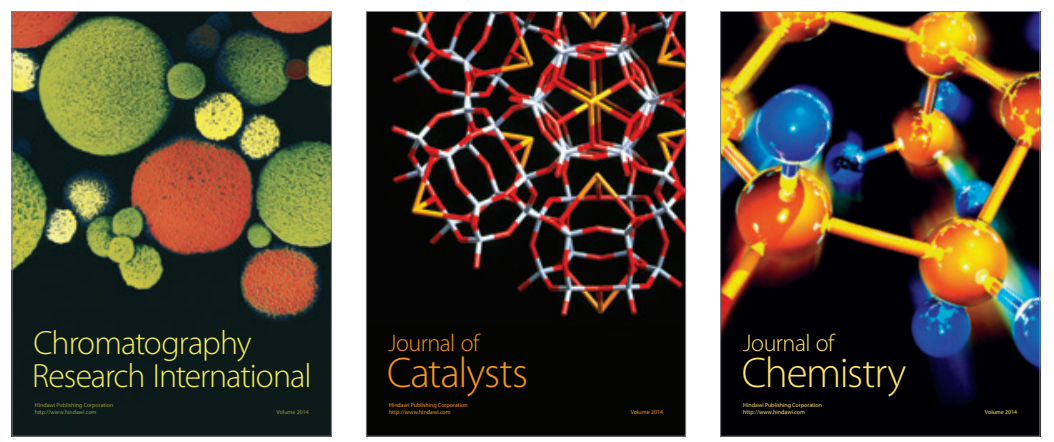
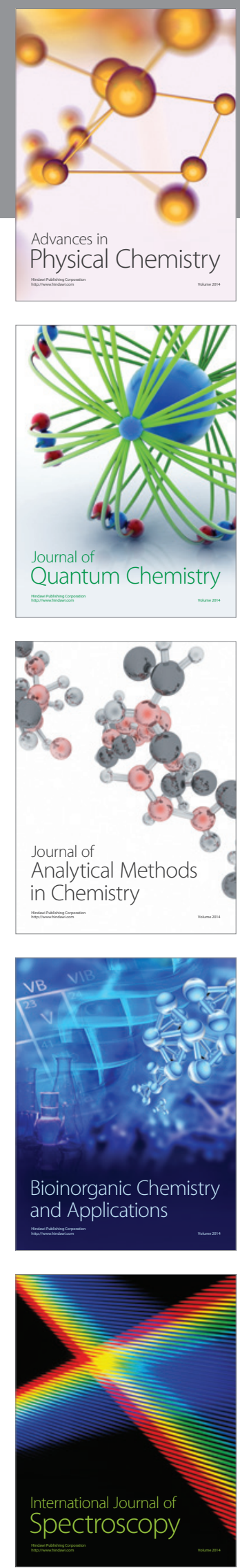